\title{
In Vivo Cancer Targeting of Water-Soluble Taxol by Folic Acid Immobilization
}

Junichi Nakamura, Naoki Nakajima, Kazuaki Matsumura and Suong-Hyu Hyon *

Department of Medical Simulation Engineering, Institute for Frontier Medical Sciences, Kyoto University, Sakyo-ku, Kyoto 606-8507, Japan

\begin{abstract}
Previously, folic acid receptor-targeted Dextran-Taxol-Folic acid (Dex-TXL-FA) has shown the in vitro superior and selective antitumor activity against human oral cancer cell line (KB) compared with the absent of folic acid, DextranTaxol. Present study is given for further investigation of in vivo antitumor efficacy of Dex-TXL-FA in the murine tumor xenograft model. To evaluate the antitumor effect of taxol, tumor bearing mice were prepared by s.c. inoculation of $1.0 \times$ $10^{6} \mathrm{~KB}$ cells in the back of nude mice. Seven days after inoculation, the administration of saline, paclitaxel for injection (PTX), Dex-TXL, FA-adsorbed Dex-TXL and Dex-TXL-FA (covalent) was started at a dose of $10 \mathrm{mg} / \mathrm{kg}$, by i.v. injection via the lateral tail vein three times ( on day 7,9 , and 11) and animal survival rate and tumor sizes were monitored.FAadsorbed Dex-TXL and Dex-TXL-FA (covalent) showed approximately 3 times greater anticancer effect than that of taxol at the 30th day after tumor implantation. Furthermore, these FA immobilized TXL showed 2-3 month longer animal survival than that of taxol. These results suggest the conjugation with Dex and FA could provide an improvement in the anticancer therapy of taxol.
\end{abstract}

\section{Introduction}

Paclitaxel is an anticancer drug used for lung, breast and ovarian tumors [1]. Due to its poor water solubility, paclitaxel is generally administered as a mixture with poly (oxyethylene) castor oil (Cremophor EL) and dehydrated ethanol [2]. Because Cremophor EL causes serious side-effects, such as irritation, in approximately $30 \%$ of patients [3], a steroid drug is required prior to its use to suppress these side effects [4]. To exclude the need for Cremophor EL, paclitaxel conjugation with poly (L-glutamic acid) [5] and albumin [6], and drug delivery systems (DDS) such as liposomes, polymer micelles, and nanoparticles have been studied to enhance its water solubility and anticancer efficacy by systematic delivery $[7,8]$. Folic acid (FA), a watersoluble vitamin that plays an important role in cell proliferation, has also been used as targeting molecule in micelle and liposome systems $[9,10]$. Overexpression of the FA receptor in some cancer cells such as ovarian and brain carcinomas [11], and a human oral cancer cell line (KB) has been reported [12].

In our previous study, the water solubility of paclitaxel and the in vitro targeting activity were remarkably enhanced by the conjugation with dextran and FA, respectively [13]. The anticancer effect was examined in vitro by using $\mathrm{KB}$ cells which overexpress $\mathrm{FA}$ receptors. FA was immobilized with dextran-conjugated paclitaxel (Dex-PXL) to provide cancer targeting to the FA receptor overexpressed on cancer cells. It was found that the water solubility of paclitaxel could be improved by dextran conjugation by as much as 2700 times, and that the cytotoxicity against KB cells could be enhanced 2-3 times by FA conjugation (Dex-PXL-FA) than that observed against a cell line without FA receptor over expression [13].

In vivo studies of DDS drugs such as micelles [14], liposomes [15], polymers [16], and nanoparticles [17] have already been reported. Recently, many studies have used antibodies [18] and peptides [19] as a ligand to target tumors. However, in spite of the specific antigen and antibody reaction for targeting, the presence of similar antigens in vivo would suppress the targeting efficacy of the drug [20]. Maeda et al. compared the permeability of immature blood vessels of cancer tissue with that of normal tissue in 1986, and they found that compounds larger than about $100 \mathrm{~nm}$ in diameter accumulated to a higher extent in cancer tissues [21]. Therefore, the drugs modified polymers to have a larger size show higher efficacy than those without the modification due to the accumulation of the larger drugs. Furthermore, these studies suggest that research should be focused on designing a drug with an enhanced permeability and retention (EPR) effect. Dextran was reported to have an EPR effect in DDS and to make a contribution to cancer-specific targeting [22]. In particular, a molecular size of 50$200 \mathrm{~nm}$ in diameter was found to be crucially important for cancer tissue targeting. Even a modified agent is immediately excreted from the kidney when the size is smaller than $50 \mathrm{~nm}$ [23]. In contrast, if the size of the conjugated drug is larger than $200 \mathrm{~nm}$, it becomes trapped in the liver and is degraded [24].

The present study further examined the in vivo antitumor efficacy of Dex-PXL-FA using a murine tumor xenograft model. In addition, the active (FA-targeting) and passive (EPR) effects of Dex-PXL-FA are also discussed in detail with regard to their molecular size evaluation.

\section{Materials and Methods}

The paclitaxel used for injection and for chemical modification were kindly donated by NIPPON KAYAKU Co., Ltd (Tokyo, Japan) and by Samyang Genex Corporation (Korea), respectively. Dextran (MW 70,000) was purchased from Meito Sangyo Co., Ltd. (Nagoya, Japan). Folic acid (FA), diethyl sulfoxide (DMSO), 1, 1 -carbonyldiimidazole (CDI), 1-ethyl-3-(3-dimethylaminopropyl) carbodiimide hydrochloride (EDC), N-hydroxysuccinimide (NHS), and ethylenediamine were purchased from Nacalai Tesque, Inc. (Kyoto, Japan) and used without further purification.

${ }^{*}$ Corresponding author: Suong-Hyu Hyon, Ph.D, Institute for Frontier Medical Sciences, Kyoto University, 53 Kawahara-cho, Shogoin, Sakyo-ku, Kyoto 6068507, Japan, Tel:. 81 757514125; Fax:. 81 757514141; E-mail: biogen@frontier. kyoto-u.ac.jp

Received December 05, 2010; Accepted January 24, 2011; Published January 24, 2011

Citation: Nakamura J, Nakajima N, Matsumura K, Hyon SH (2011) In Vivo Cancer Targeting of Water-Soluble Taxol by Folic Acid Immobilization. J Nanomedic Nanotechnol 2:106. doi:10.4172/2157-7439.1000106

Copyright: (c) 2011 Nakamura J, et al. This is an open-access article distributed under the terms of the Creative Commons Attribution License, which permits unrestricted use, distribution, and reproduction in any medium, provided the original author and source are credited. 


\section{Cell culture}

KB cells (DS Pharma Biomedical Co., Ltd., Osaka, Japan) were cultured in RPMI1640 (folate-free, Invitrogen Japan, Tokyo, Japan) supplemented with $10 \%$ fetal bovine serum and $100 \mathrm{~g} / \mathrm{ml}$ of penicillinstreptomycin at $37^{\circ} \mathrm{C}$ under $5 \% \mathrm{CO}_{2}$ in a humidified atmosphere. When the cells reached $80 \%$ confluence, they were detached by $0.25 \%(\mathrm{w} / \mathrm{v})$ of trypsin containing $0.02 \%(\mathrm{w} / \mathrm{v})$ of ethylenediamine tetracetic acid (EDTA) in phosphate buffered saline without calcium and magnesium (PBS(-)) and seeded on a new tissue cuture plate for subculture. The KB cells were used for each experiment within five passages.

\section{Synthesis of amino-Dex, Dex-PXL, FA-adsorbed Dex-PXL and Dex-PXL-FA (covalent)}

Dex-PXL, FA-adsorbed Dex-PXL, and Dex-PXL-FA (covalent) were synthesized according to the previous report [13]. Briefly, synthesis method of their complex was shown as follows. Dextran (10 g) was dissolved in $70 \mathrm{ml}$ DMSO and mixed with a solution of $2 \mathrm{~g}$ CDI in $5 \mathrm{ml} \mathrm{DMSO}$, and the activation reaction was preceded at $50^{\circ} \mathrm{C}$ for 15 min. Subsequently, $5 \mathrm{ml}$ of ethylenediamine was added and the mixture was stirred at $50^{\circ} \mathrm{C}$ for $18 \mathrm{~h}$. After dialysis against running water for 24 $\mathrm{h}$ and distilled water $(3 \mathrm{~L}, 1.5 \mathrm{~h} \times 2)$ with a dialysis membrane (cut-off molecular weight of 14,000 Da), amino-Dex was recovered by air and vacuum drying. To activate the paclitaxel $\mathrm{OH}$ group, a solution of $0.7 \mathrm{~g}$ paclitaxel in $40 \mathrm{ml}$ DMSO was added to a solution of $0.6 \mathrm{~g}$ CDI dissolved in $10 \mathrm{ml} \mathrm{DMSO}$ at $50^{\circ} \mathrm{C}$ for $15 \mathrm{~min}$. Five grams of amino-Dex in $150 \mathrm{ml}$ of DMSO was added to the solution, and the reaction proceeded at $50^{\circ} \mathrm{C}$ for $18 \mathrm{~h}$. Dex-PXL was recovered by the same purification manner as described above. The yield of Dex-PXL was about 70. Recovered DexPXL and FA were dissolved in PBS at a concentration of 800 and $240 \mathrm{~g} /$ $\mathrm{ml}$, respectively. Here, a solution of $0.5 \mathrm{ml}$ Dex-PXL was simply mixed with $0.5 \mathrm{ml}$ of FA and stirred at $25^{\circ} \mathrm{C}$ for $18 \mathrm{~h}$ to prepare FA-adsorbed Dex-TXL. A solution of $0.1 \mathrm{~g}$ FA and $0.15 \mathrm{~g}$ NHS in $20 \mathrm{ml}$ DMSO was mixed with $0.07 \mathrm{~g}$ of EDC in $10 \mathrm{ml}$ DMSO, and the activation reaction of the FA COOH groups proceeded at $50^{\circ} \mathrm{C}$ for $5 \mathrm{~min}$. Then, $0.5 \mathrm{~g}$ of Dex-PXL in $10 \mathrm{ml}$ DMSO was added to the mixture and reacted at $50^{\circ} \mathrm{C}$ for $3 \mathrm{~h}$. Dex-PXL-FA (covalent) was recovered after purification by dialysis against water and drying.

\section{Molecular size evaluation by dynamic light scattering (DLS)}

The hydrodynamic molecular size of the conjugates was measured at $25^{\circ} \mathrm{C}$ by DLS using a DLS-7000 instrument (Otsuka Electronics, Osaka, Japan) equipped with a He-Ne laser. The samples were dissolved in PBS and DMSO (for paclitaxel) at a concentration of 5000ppm, and $5 \mathrm{ml}$ of the solution was used for the evaluation.

\section{Animals}

Female nude mice (BALB/c nu/nu; 4weeks old and weighing 9-17 g) were purchased from Charles River Laboratories Japan, Inc (Kanagawa, Japan). The nude mice were maintained on a folate-free rodent diet (AIN-93G-based Folate-Deficient Rodent Diet, Oriental Yeast CO., LTD, Tokyo, Japan) on arrival and for the duration of the study. Animal experiments were performed according to the criteria established in the Guidelines of the Committee on Animal Care and Use of Kyoto University.

\section{Acute toxicity}

To evaluate the acute toxicity of the conjugates, mice were administered a single i.v. injection of $100 \mu \mathrm{l} /$ mouse containing 140 $220 \mu \mathrm{g}$ of paclitaxel. The body weights of mice were recorded for 2 weeks and compared with those of tumor-free nude mice (6 weeks old and weighting 14-22g).

\section{Antitumor effects and survival rates in tumor-bearing mice}

To evaluate the antitumor effect of paclitaxel, tumor-bearing mice were prepared by inoculating KB cells S.C onto the back of nude mice $\left(1.0 \times 10^{6}\right.$ cells/mouse $)$. Seven days after inoculation, paclitaxel for injection (PXL), Dex-PXL, FA-adsorbed Dex-PXL, and Dex-PXL-FA (covalent) were administrated by i.v. injection via the lateral tail vein three times (at a dose of $10 \mathrm{mg}$ PXL $/ \mathrm{kg}$, on days 7,9 , and 11 ). Tumor size was measured with a digital vernier caliper and the volume was calculated using the formula for a prolate ellipsoid, $\mathrm{V}\left(\mathrm{mm}^{3}\right)=(\mathrm{a} \times$ $\left.b^{2}\right) / 2$, where $a$ is the longer, and $b$ is the shorter diameter. The survival rate was also monitored at 2 or 3 day intervals.

\section{Tumor-targeting effect of Dex-PXL-FA}

To investigate the tumor-targeting effect of $\mathrm{FA}$, fluorescein isothiocyanate (FITC)-labelled FA-adsorbed Dex-PXL (Dex-PXLFITC) was prepared as follows: In one glass vial, Dex-PXL and FITC were dissolved in DMSO at the concentration of $10 \mathrm{w} / \mathrm{v}$ and $0.075 \mathrm{w} / \mathrm{v}$, respectively, and stirred at $50^{\circ} \mathrm{C}$ for $1 \mathrm{~h}$, followed by re-precipitation of Dex-PXL-FITC with an excess of acetone and vacuum drying. The recovered Dex-PXL-FITC and FA were dissolved in PBS at concentrations of 800 and $240 \mu \mathrm{g} / \mathrm{ml}$, respectively, and $0.5 \mathrm{ml}$ of each solution was simply mixed and stirred at $25^{\circ} \mathrm{C}$ for $18 \mathrm{~h}$ to prepare $\mathrm{FA}$ adsorbed Dex-PXL-FITC (Dex-PXL-FITC-FA). The targeting effect was evaluated using the same method as that for the antitumor effects. The tumor tissue was isolated from each mouse on the day after the third injection. Tumor tissues were bisected and placed in the TissueTek $^{\otimes}$ Optimum Cutting Temperature compound (Sakura Ltd. Tokyo, Japan) for cryosection, and the distribution of Dex-PXL-FITC-FA was observed under an optical/fluorescence microscope (Biozero-8000, Keyence, Osaka, Japan).

\section{Statistical analyses}

All data are shown as the means \pm standard deviation (SD). Data among the groups were compared by Tukey-Kramer multiple comparison test. Differences were considered to be statistically significant at $\mathrm{p}<0.05$.

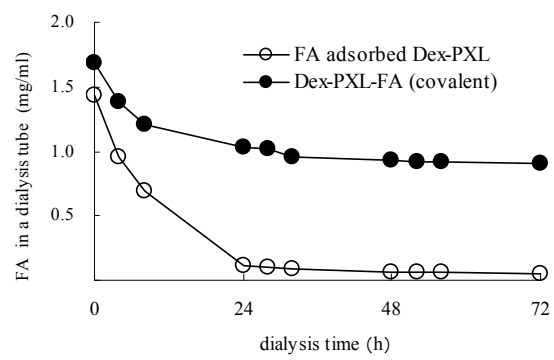

Figure 1: Changes in the FA concentration in the dialysis tube by dialysis against $\mathrm{PBS}$ at $25^{\circ} \mathrm{C}$

\begin{tabular}{|l|l|}
\hline Sample & Molecular size $(\mathrm{nm})$ \\
\hline Paclitaxel & $11.2 \pm 1.2$ \\
\hline Dextran & $14.2 \pm 3.8$ \\
\hline amino-Dex & $18.5 \pm 1.0$ \\
\hline Dex-PXL & $97.1 \pm 2.2$ \\
\hline FA-adsorbed Dex-PXL & $93.0 \pm 3.2$ \\
\hline Dex-PXL-FA (covalent) & $89.8 \pm 7.1$ \\
\hline *Only taxol was dissolved in DMSO and the others were in PBS \\
\multicolumn{2}{|l|}{ Table 1: Average size of samples by DLS measured. $\left(5000 \mathrm{ppm}, 25^{\circ} \mathrm{C}\right)}$. \\
\hline
\end{tabular}




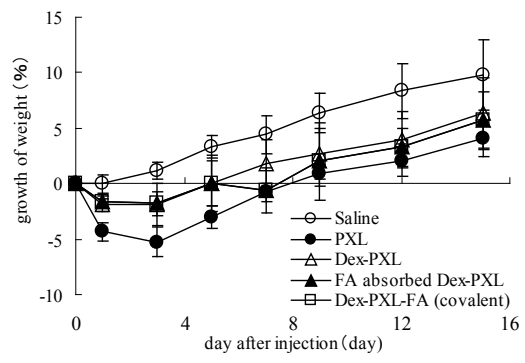

Figure 2: Body weight changes after injection of the conjugates. Female nude mice were given a single i.v. injection of saline, PXL, Dex-PXL, FA-adsorbed Dex-PXL, or Dex-PXL-FA (covalent). Data are the average \pm S.D $(n=10)$.

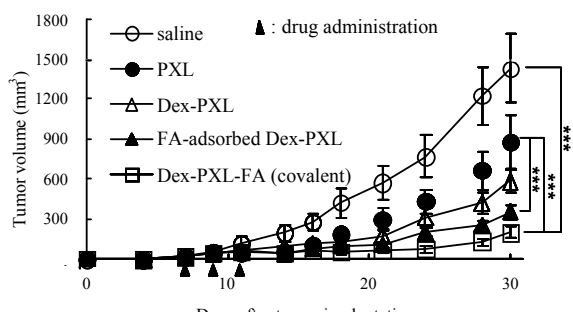

Days after tumor implantation

Figure 3: Tumor growth inhibition by PXL, Dex-PXL, FA-adsorbed Dex-PXL, and Dex-PXL-FA (covalent). Nude mice with KB xenograft tumors were treated with a series of three i.v. injections (given every other day, as indicated by the arrow heads) of Dex-PXL containing $10 \mathrm{mg} / \mathrm{kg} \mathrm{PXL} \mathrm{(arrow} \mathrm{head} \mathrm{showing} \mathrm{the}$ day of administration). ${ }^{* *}$ means $p<0.001$.

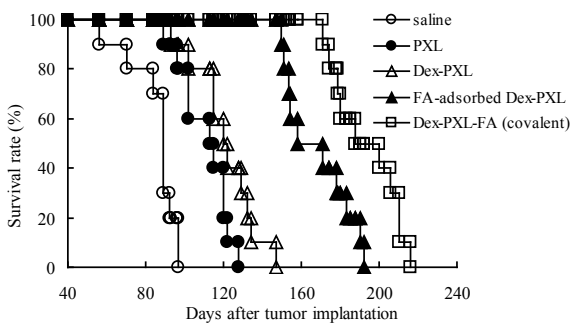

Figure 4: The survival rate of nude mice bearing KB tumors. The mice $(n=10 /$ group) were given three i.v. injections, then the tumor volume was evaluated every other day. Saline vs. PXL $P=0.0008$; PXL vs. Dex-PXL $P=0.07$; PXL vs. FA-adsorbed Dex-PXL $P=0.0002$; PXL vs. Dex-PXL-FA (covalent) $P=$ 0.0002 .
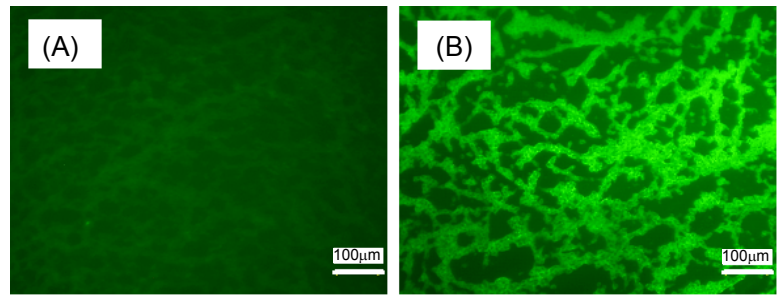

Figure 5: Fluorescence microphotographs of cryosections of tumor tissues. Dex-PXL-FITC (A) and FA-adsorbed Dex-PXL-FA (B). The exposure time was $10 \mathrm{sec}$ in both cases.

\section{Results}

\section{FA-conjugation with Dex-PXL}

The results of the PBS dialysis of Dex-PXL-FA (covalent) and FA- adsorbed Dex-PXL are shown in Figure 1. In the case of FA-adsorbed Dex-PXL, FA easily detached from the Dex-PXL during PBS dialysis, and FA almost completely disappeared within 24 hours. In contrast, the extent of FA decrease from Dex-PXL-FA (covalent) was far smaller and $60 \%$ of the FA remained after $72 \mathrm{~h}$, confirming the covalent immobilization of FA into Dex-PXL.

\section{Molecular size evaluation by DLS}

The average molecular size of each sample measured by DLS is summarized in Table 1. The molecular size of paclitaxel in DMSO and dextran and amino-Dex in PBS were closely arranged, within 10-20 nm. In contrast, Dex-PXL, FA-adsorbed Dex-PXL and Dex-PXL-FA (covalent) were much larger. After paclitaxel conjugation in dextran, the particle size became about 10 times larger due to high hydrophobicity of paclitaxel molecules. The particle size of Dex-PXL, FA-adsorbed Dex-PXL, and Dex-PXL-FA (covalent) suggested an EPR effect in vivo.

\section{Acute toxicity}

Each sample was administered to intact mice (nude mouse $\mathrm{n}=5$ ) as a single i.v. injection, and the body weight change was recorded for two weeks. The results are given in Figure 2. The body weight of saline-treated mice increased normally. On the other hand, the weight decreased until the third day, when PXL was administered. Dex-PXL, FA-adsorbed Dex-PXL and Dex-PXL-FA (covalent) led to a smaller weight loss than PXL.

\section{Antitumor effects and survival of tumor-bearing mice}

The tumor growth inhibitory activities were evaluated using KB xenograft tumor-bearing nude mice. As shown in Figure 3, the tumors rapidly grew in size when the mice were treated with saline. PXL controlled cancer growth better than saline, and showed remarkable cancer growth inhibition after the 20th day. After the 30th day, the mean tumor volumes in the PXL and Dex-PXL groups were 600 and $900 \mathrm{~mm}^{3}$, respectively. FA-adsorbed Dex-PXL and Dex-PXL-FA (covalent) further delayed the tumor growth, and almost complete growth suppression was observed around 3 weeks, which suggested an excellent targeting effect of FA.

The effects of the drugs on for the survival of tumor-bearing mice were also evaluated, and the survival data are summarized in Figure 4. The average survival of the saline, PXL, Dex-PXL, FA-adsorbed Dex-PXL and Dex-PXL-FA (covalent) treated mice were $84.7 \pm 12.3$, $110.7 \pm 12.7,120.9 \pm 15.8,168.3 \pm 16.8$ and $193.4 \pm 16.8$ days, respectively. The mice treated with PXL and Dex-PXL showed almost the same survival rate. In contrast, far longer survival (2-3 months) was observed in FA-adsorbed Dex-PXL and Dex-PXL-FA (covalent)-treated mice.

\section{FA targeting effect using FA-adsorbed Dex-PXL-FITC}

The targeting effect of the FA-adsorbed Dex-PXL was evaluated by FITC conjugation, and the results are shown in Figure 5. Figures $5 \mathrm{~A}$ and $\mathrm{B}$ show the fluorescent microscopic images of the cancer tissue 12 days after injection of Dex-PXL-FITC and FA-adsorbed DexPXL-FITC, respectively. The fluorescence intensity of Dex-PXL-FITC injected tissue was higher than that of Dex-PXL-FITC, thus suggesting the better targeting effect of FA conjugation.

\section{Discussion}

In the present study, we evaluated the in vivo anticancer effects of water soluble and FA immobilized paclitaxel conjugations. DexPXL without FA conjugation showed an improved anticancer effect 
Citation: Nakamura J, Nakajima N, Matsumura K, Hyon SH (2011) In Vivo Cancer Targeting of Water-Soluble Taxol by Folic Acid Immobilization. J Nanomedic Nanotechnol 2:106. doi:10.4172/2157-7439.1000106

Page 4 of 4

compared to PXL, which was due to the EPR effect of the molecule. In addition, the anticancer effect was remarkably enhanced by FA immobilization to Dex-PXL. Both FA-adsorbed and FA-covalently bound Dex-PXLs exerted enhanced antitumor activity against FA receptor over expressing cancer cells [13].

It is likely that the FA immobilized by adsorption might be desorbed into the systemic circulation in vivo, and Figure 1 suggests that there is a loss of the anticancer efficacy of FA-adsorbed Dex-PXL during PBS dialysis compared with Dex-PXL-FA (covalent). However, Figure 5B clearly showed that the FA-adsorbed Dex-PXL-FITC remained in the tumor tissue even 11 days after injection, suggesting the rapid accumulation of FA-adsorbed Dex into the tissue before FA desorption. This is in agreement with the fact that the tumor volume in mice treated with FA-adsorbed Dex-PXL after 30 days was smaller than that of Dex-PXL (Figure 4). These results reveal the usefulness of the easy adsorption method for targeting. The Dex-PXLs with FA immobilized by both covalent bonding and adsorption suppressed the tumor growth, however, the survival rate was significantly higher when covalently bonded drug was selected.

The FA receptor is abundantly expressed in a large percentage of human tumors, but it is only minimally distributed in a normal tissue [25-28]. The FA receptor has three isoforms $\alpha, \beta$ and $\gamma$. The isoform is expressed in some normal epithelial cells, and its expression is elevated in certain carcinomas [23]. It was reported that KB [29, 30], coco2 [31], and ovarian carcinomas cells express the FA receptor $\alpha$ on their surface. Therefore, FA-adsorbed and FA-covalently bound Dex-PXL are excellent tumor targeting agents for cells expressing FA receptor $\alpha$.

In summary, FA-adsorbed Dex-PXL and Dex-PXL-FA (covalent) showed excellent anticancer effects due to their EPR and cancer targeting effects, with the conjugation of FA and Dex providing more effective and safer anticancer therapy with paclitaxel than when the agent is used alone.

\section{Acknowledgements}

The authors gratefully express their appreciation to Samyang Genex Corporation, Korea and NIPPON KAYAKU Co., Ltd, Tokyo, Japan for the kindly supplying us with paclitaxel.

\section{References}

1. Ramesh P (1998) Pharmaceutical aspects of Paclitaxel. Int J Pharm 172: 1-15.

2. Adams JD, Flora KP, Goldspiel BR, Wilson JW, Arbuck SG, et al. (1993) Taxol: a history of pharmaceutical development and current pharmaceutical concerns. J Natl Cancer Inst Monogr 15: 141-147.

3. Wang J, Mongayt D, Torchilin VP (2005) Polymeric micelles for delivery of poorly soluble drugs: preparation and anticancer activity in vitro of paclitaxe incorporated into mixed micelles based on poly(ethylene glycol)-lipid conjugate and positively charged lipids. J Drug Target 13: 73-80.

4. Rowinsky EK, McGuire WP, Guarnieri T, Fisherman JS, Christian MC, et al (1991) Cardiac disturbances during the administration of taxol. J Clin Oncol 9 : 1704-1712.

5. Chun L, Dong-Fang Y, Robert AN, Fernando C, L Clifton S, et al. (1998) Complete regression of well-established tumors using a novel water-soluble poly (L-glutamic acid)-paclitaxel conjugate. Cancer Res 58: 2404-2409.

6. William JG (2006) Albumin-bound paclitaxel: a next-generation taxane. Expert Opin Pharmacother 7: 1041-1053.

7. Xiang G, Wu J, Lu Y, Liu Z, Lee RJ (2008) Synthesis and evaluation of a nove ligand for folate-mediated targeting liposomes. Int J Pharm 356: 29-36.

8. Bae Y, Nishiyama N, Kataoka K (2007) In vivo antitumor activity ofthe folateconjugated $\mathrm{pH}$-sensitive polymeric micelle selectively releasing adriamycin in the intracellular acidic compartments. Bioconjug Chem 18: 1131-1139.

9. Seow WY, Xue JM, Yang YY (2007) Targeted and intracellular delivery of paclitaxel using multi-functional polymeric micelles. Biomaterials 28: 1730 1740

10. Wu J, Liu Q Lee RJ (2006) A folate receptor-targeted liposomal formulation for paclitaxel: Int J Pharm 316: 148-153.

11. Lu Y, Low PS (2003) Immunotherapy of folate receptor-expressing tumors: review of recent advances and future prospects. J Control Release 91: 17-29.

12. Weitman SD, Lark RH, Coney LR, Fort DW, Frasca V (1992) Distribution of the folate receptor GP38 in normal and malignant cell lines and tissues. Cancer Res 52: 3396-3401.

13. Nakamura J, Nakajima N, Matsumura K, Hyon SH (2010) Water-soluble taxol conjugates with dextran and targets tumor cells by folic acid immobilization. Anticancer Res 30: 903-909.

14. Maeda H, Sawa T, Konno T (2001) Mechanism of tumor-targeted delivery of macromolecular drugs, including the EPR effect in solid tumor and clinical overview of the prototype polymeric drug SMANCS. J Control Release 74: 47-61.

15. Schwendener RA (2007) Liposomes in biology and medicine. Adv Exp Med Biol 620: 117-128.

16. Shibata H, Nakagawa S, Tsutsumi $Y$ (2005) Optimization of protein therapies by polymer-conjugation as an effective DDS. Molecules 31: 162-180.

17. Nassimi M, Schleh C, Lauenstein HD, Hussein R, Lubbers K (2009) Low cytotoxicity of solid lipid nanoparticles in vitro and ex vivo lung models. Inhal Toxicol 21: 104-109.

18. Tsutsui $Y$, Tomizawa K, Nagita M, Michiue H, Nishiki T (2007) Development of bionanocapsules targeting brain tumors. J Control Release 26: 159-164

19. Yu DH, Lu Q, Xie J, Fang C, Chen HZ (2010) Peptide-conjugated biodegradable nanoparticles as a carrier to target paclitaxel to tumor neovasculature. Biomaterials 31: 2278-2292.

20. Abu-hadid MM, Bankert RB Mayers GL (1987) Antigen-specific drug-targeting used to manipulate an immune response in vivo. Proc Natl Acad Sci USA 84: $7232-7236$.

21. Matsumura $Y$, Maeda $H$ (1986) A new concept for macromolecular therapeutics in cancer chemotherapy: mechanism of tumoritropic accumulation of proteins and the antitumor agent smancs. Cancer Res 46: 6387-6392.

22. Bisht S, Maitra A (2009) Dextran-doxorubicin chitosan nanoparticles for solid tumor therapy. Wiley Interdiscip Rev Nanomed Nanobiotechnol 1: 415-425.

23. Chisholm EJ, Vassaux G, Martin-Duque P, Chevre R, Lambert O, et al. (2009) Cancer-specific transgene expression mediated by systemic injection of nanoparticles. Cancer Res 69: 2655-2662.

24. Adiseshaiah PP, Hall JB, McNeil SE (2010) Nanomaterial standards for efficacy and toxicity assessment. Wiley Interdiscip Rev Nanomed Nanobiotechnol 2 99-112.

25. Wu M, Gunning W, Ratnam M (1999) Expression of folate receptor type a in relation to cell type, malignancy, and differentiation in ovary, uterus, and cervix. Cancer Epidemiol Biomarkers Prev. 8: 775-782.

26. Shen F, Ross JF, Wang X, Ratnam M (1994) Identification of a novel folate receptor, a truncated receptor, and receptor type $\beta$ in hematopoietic cells: cDNA cloning, expression, immunoreactivity, and tissue specificity. Biochemistry 33 1209-1215.

27. Ross JF, Wang H, Behm FG, Mathew P, Wu M (1999) Folate receptor type $\beta$ is a neutrophilic lineage marker and is differentially expressed in myeloid leukemia. Cancer 85: 348-357.

28. Wang H, Ross JF, Ratnam M (1998) Structure and regulation of a polymorphic gene encoding folate receptor type $Y / Y^{\prime}$. Nucleic Acids Res 26: 2132-2142.

29. Lee RJ, Low PS. (1995) Folate-mediated tumor cell targeting of liposomeentrapped doxorubicin in vitro. Biochim Biophys Acta 1233: 134-144.

30. Zhai G, Wu J, Xiang G, Mao W, Yu B (2009) Preparation, characterization and pharmacokinetics of folate receptor-targeted liposomes for docetaxel delivery. J Nanosci Nanotechnol 9: 2155-2161

31. Chattopadhyay S, Wang Y, Zhao R, Goldman ID (2004) Lack of impact of the loss of constitutive folate receptor alpha expression, achieved by RNA Interference, on the activity of the new generation antifolate pemetrexed in HeLa cells. Clin Cancer Res 10: 7986-7993. 